\title{
Controller design for underwater robotic vehicle based on improved whale optimization algorithm
}

\author{
Mustafa Wassef Hassan, Nizar Hadi Abbas \\ Department of Electrical Engineering, University of Baghdad, Iraq
}

\begin{tabular}{l} 
Article Info \\
\hline Article history: \\
Received Jul 12, 2020 \\
Revised Nov 25, 2020 \\
Accepted Jan 12, 2021 \\
\hline
\end{tabular}

\section{Keywords:}

AUV

FOPID

Improved WOA

LQG

PID

\begin{abstract}
This paper presents the impact of introducing a two-controller on the linearized autonomous underwater vehicle (AUV) for vertical motion control. These controllers are presented to overcome the sensor noise of the AUV control model that effect on the tolerance and stability of the depth motion control. Linear quadratic Gaussian (LQG) controller is cascaded with AUV model to adapt the tolerance and the stability of the system and compared with FOPID established by the improved whale optimization algorithm (IWOA) to identify which controlling method can make the system more harmonize and tolerable. The developed algorithm is based on improving the original WOA by reshaping a specific detail on WOA to earn a warranty that the new IWOA will have values for the update position lower than the identified lower-bound (LB), and upper-bound (UB). Furthermore, the algorithm is examined by a set of test functions that include (unimodal, multimodal and fixed dimension multimodal functions). The privileges of applying IWOA are reducing the executing time and obtaining the semioptimal objective function as compared with the original WOA algorithm and other popular swarm-intelligence optimization algorithms.
\end{abstract}

This is an open access article under the CC BY-SA license.

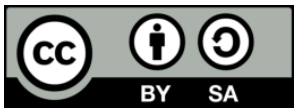

\section{Corresponding Author:}

Mustafa Wassef Hasan

Department of Electrical Engineering

University of Baghdad

Al-Jadriya, Baghdad, Iraq

Email: m.hasan0902@coeng.uobaghdad.edu.iq

\section{INTRODUCTION}

Autonomous underwater vehicle (AUV) considered one of the several studies that have discussed during the last decades due to its purposes and applications in different fields such as militaries, exploring the ocean submarines, oil and gas fields. In the literature, numerous controllers are presented for control the AUV the authors suggested different techniques to solve or handle the AUV problems like [1-5]. During the movement of AUV towards the vertical location, the sensors used in the AUV control model can add a considerable amount of noise which leads to change the tolerance and stability of the system as a result of that movement of AUV become unsettled. To solve this problem LQG controller used in compared with FOPID based on basic WO and IWO algorithms, which considered one of many swarm-intelligence algorithms that used to enhance selected system controllers by getting the best tuning parameters for the running algorithm like [6] that achieved the global time optimization by introducing quantum behaviour and adaptive law into a particle optimization algorithm (PSO), the authors in [7] explain a nature-inspired algorithm called alarm pheromone ant colony system (AP-ACS) in order to handle the constraint issue for the path planning effectively.

Therefore, to present the capability of the proposed IWO algorithm that developed based on the definition of the basic WO algorithm, several test functions are utilized to obtain the average and standard 
deviation and finally to be compared with other optimization algorithms [8-10]. The remainder of this is organized as follows. Section 2 establishes the autonomous underwater vehicle model. The theoretical basics for the controlling methods and the standard whale optimization algorithm are presented in section 3 . The improvement of the standard WOA is explored fully in section 4. Section 5 describes the numerical results of the improved WOA capability in comparison with the basic one and the results of the system with designed controllers tuned by different optimization algorithms. Finally, section 6 summarizes the conclusions of this research work.

\section{AUTONOMOUS UNDERWATER VEHICLE MODEL}

Model design of the AUV [11, 12] presented as body-fixed reference (BRF) and inertial reference frame (IRF) as shown in Figure 1. AUV dynamics described by vector velocity $v=[u, v, w, p, q, r]^{T}$ which represent linear velocities and angular velocities of (Surge, Sway, Heave, Roll, Pitch, Yaw) respectively, while IRF can express as the vector $\eta=\left[\eta_{1}, \eta_{2}\right]^{T}$ where $\eta_{1}=[x, y, z]^{T}$ and $\eta_{2}=[\varphi, \theta, \psi]^{T}$ both $\eta_{1}$ and $\eta_{2}$ represents the position and rotational coordinate of AUV.

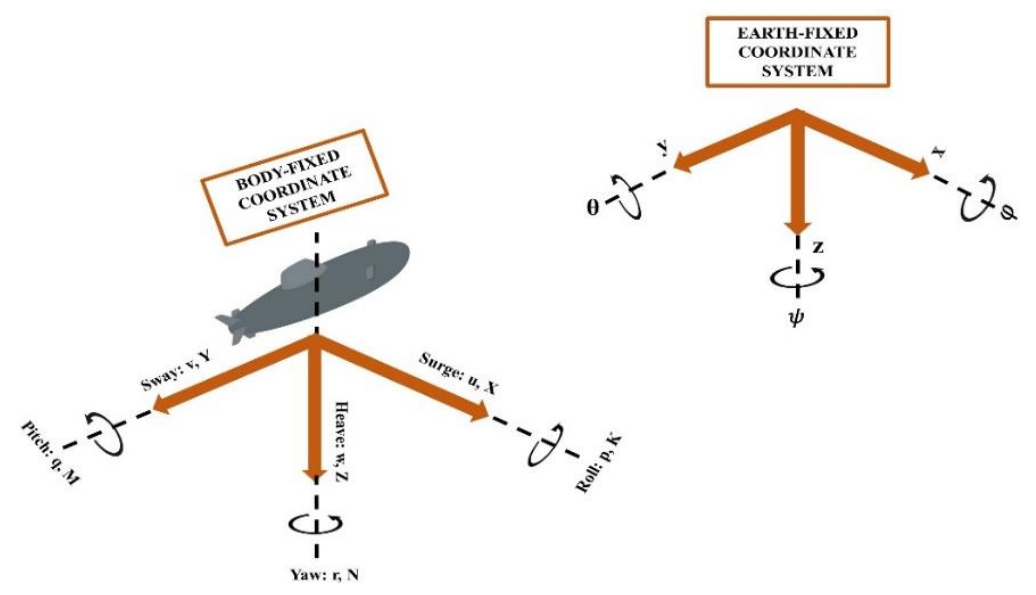

Figure 1. AUV reference frames

The author in [13] described vehicle dynamics as (1):

$$
\begin{aligned}
& m\left[\dot{u}-v r+w q-x_{g}\left(q^{2}+r^{2}\right)+y_{g}(p q-\dot{r})+z_{g}(p r+\dot{q})\right]=\sum X_{\text {ext }} \\
& m\left[\dot{v}-w p+u r-y_{g}\left(r^{2}+p^{2}\right)+z_{g}(q r-\dot{p})+x_{g}(q p+\dot{r})\right]=\sum Y_{\text {ext }} \\
& m\left[\dot{w}-u q+v p-z_{g}\left(p^{2}+q^{2}\right)+x_{g}(r p-\dot{q})+y_{g}(r q+\dot{p})\right]=\sum Z_{\text {ext }} \\
& I_{x x} \dot{p}+\left(I_{z z}-I_{y y}\right) q r+m\left[y_{g}(\dot{w}-u q+v p)-z_{g}(\dot{v}-w p+u r)\right]=\sum K_{\text {ext }} \\
& I_{y y} \dot{q}+\left(I_{x x}-I_{y y}\right) r p+m\left[z_{g}(\dot{u}-v r+w q)-x_{g}(\dot{w}-u q+v p)\right]=\sum M_{\text {ext }} \\
& I_{z z} \dot{r}+\left(I_{y y}-I_{x x}\right) p q+m\left[x_{g}(\dot{v}-w p+u r)-y_{g}(\dot{u}-v r+w q)\right]=\sum N_{\text {ext }}
\end{aligned}
$$

where the parameters of the vehicle dynamics of equations above are, $(\mathrm{x})$ is the forward position, (y) is the backwards position, $(\mathrm{z})$ is the depth position, $(\phi)$ is the roll angle, $(\theta)$ is the pitch angle, $(\psi)$ is yaw angle, $(\mathrm{m})$ is the mass of the vehicle, $\left(X_{\text {ext }}, Y_{\text {ext }}, Z_{\text {ext }}, K_{\text {ext }}, M_{\text {ext }}, N_{\text {ext }}\right)$ are the Total forces and moments acting on the vehicle to the body-fixed reference frame, $\left(I_{x x}, I_{y y}, I_{z z}\right)$ are the inertia tensor, $\left(x_{g}, y_{g}, z_{g}\right)$ are the vehicle gravity coordinates, and $\left(x_{b}, y_{b}, z_{b}\right)$ are the buoyancy coordinates of the AUV.

Vehicle mechanics can be linearized and developing the state-space matrices as described below,

$$
\left[\begin{array}{cccc}
m-X_{\dot{u}} & -\left(m X_{g}+Z_{\dot{q}}\right) & 0 & 0 \\
-\left(m X_{g}-M_{\dot{w}}\right) & I_{y y}-M_{\dot{q}} & 0 & 0 \\
0 & 0 & 1 & 0 \\
0 & 0 & 0 & 1
\end{array}\right]\left[\begin{array}{c}
\dot{W} \\
\dot{q} \\
\dot{Z} \\
\dot{\theta}
\end{array}\right]-\left[\begin{array}{cccc}
Z_{w} & m U+Z_{q} & 0 & 0 \\
M_{w} & -m X_{g} U+M_{q} & 0 & M_{\theta} \\
1 & 0 & 0 & -U \\
0 & 1 & 0 & 0
\end{array}\right]\left[\begin{array}{c}
w \\
q \\
z \\
\theta
\end{array}\right]=\left[\begin{array}{c}
Z \delta_{s} \\
M \delta_{s} \\
0 \\
0
\end{array}\right]\left[\delta_{s}\right]
$$


where $u=\left[\delta_{s}\right]^{T}$, which $\left(\delta_{s}\right)$ the AUV plane angle, (2) can be written as,

$$
\dot{x}=A x+B u
$$

After developing the values of the parameters in [13] in (3), the following parameters are produced:

$$
A=\left(\begin{array}{cccc}
-2.38 & 1.26 & 0 & 0.04 \\
4.23 & -1.12 & 0 & -0.7 \\
1 & 0 & 0 & -1.54 \\
0 & 1 & 0 & 0
\end{array}\right), B=\left(\begin{array}{c}
-1.38 \\
-3.84 \\
0 \\
0
\end{array}\right) \& C=\left(\begin{array}{llll}
0 & 0 & 1 & 0
\end{array}\right)
$$

\section{THEORETICAL BASICS}

\subsection{LQG controller}

Linear quadratic Gaussian (LQG) controller is a mixed between Kalman filter and linear quadratic regulator (LQR) controller [14], that used for finding the optimal parameters" values that minimizing the quadratic cost criterion. Kalman filter is known as an estimator for a linear-quadratic problem which responsible for estimating the system states when additive white noise added to the linearized system's model. Where the system state equation defined as;

$$
\begin{aligned}
& \dot{X}_{(t)}=A X_{(t)}+B u_{(t)}+\Gamma w_{(t)} \\
& y_{(t)}=C X_{(t)}+v_{(t)}
\end{aligned}
$$

where $X_{(t)}$ is the state vector, $v_{(t)}$ and $w_{(t)}$ are the additive white noise signals, $\Gamma$ is the disturbance matrix, $u_{(t)}$ is the control input which used to minimize the performance index shown in (6) and $y_{(t)}$ is the system output.

$$
J=E\left\{\lim _{T \rightarrow \infty} \frac{1}{T} \int_{0}^{T}\left[x^{T} Q x+u^{T} R u\right] d t\right\}
$$

where $\mathrm{Q}$ and $\mathrm{R}$ are weighting matrices such that $\mathrm{Q}=Q^{T} \geq 0$ and $R=R^{T}>0, \mathrm{E}$ is the expected value. The linear state-space described as:

$$
\begin{aligned}
& \dot{X}_{(t)}=A X_{(t)}+B u_{(t)} \\
& u(t)=-K_{r} X_{(t)}
\end{aligned}
$$

where $\mathrm{Kr}$ is obtained from the performance index represented in (6);

$$
K_{r}=R^{-1} B^{T}
$$

where $P$ can be found from solving the matrix Riccati equation (MRE). Kalman filter matrix also can be found from (10),

$$
K_{f}=P_{n} C^{-1} v^{-1}
$$

where $P_{n}$ can be found from solving the following matrix Riccati equation

$$
A P_{n}+P_{n} A^{T}+\Gamma W^{T} \Gamma-P_{n} C^{T} v^{-1} C P_{n}=0
$$

\subsection{Luenberger observer}

Luenberger observer is used in different models such as discrete-time observer, continuous-time model, nonlinear observer, and others. Luenberger observer used to estimate states in real systems by measure input and output of these systems, and in some cases, it is necessary to find the system states when it involved with other sources such as adding additive white noise to the system and because of that, the system will not solve this issue correctly when noise added for that reason, the observer is designed to define the real states of such systems [15]. 
A model is constructed, but first, the system should be tested to ensure it is fully observable using the following formula, $Q_{o}=\left[\begin{array}{lll}C & C A C A^{2} \ldots C A^{n-1}\end{array}\right]$, so that it should have full rank similar to state matrix $(A)$ to estimate all state variables as shown in the following equations:

$$
\begin{aligned}
& K_{f}=P_{n} C^{-1} v^{-1} \\
& \dot{\hat{X}}=A \hat{x}+B u
\end{aligned}
$$

where $\dot{\hat{X}}$ is the estimation of real states, (13) can be written as shown below,

$$
\dot{\hat{X}}=A \hat{x}+B u+L(y-\hat{y})
$$

After adding the difference between the measured and the estimation output multiplied by $(L)$, i.e., $L(y-\hat{y})$ which be considered as convergence term to the system. The error of the system can be described in the following,

$$
\dot{X}-\dot{\hat{X}}=A(x-\hat{x})-L(C x-C \hat{x})
$$

The value of $L$ must be found in order to stabilize the system, it is normally assumed arbitrary but preferred as 5 times of closed-loop poles of the system.

\subsection{FOPID controller}

Fractional order proportional, integral and derivative controller consists of five parameters that different from typical PID controller, which normally consists of three parameters to enlarge the search space and therefore, achieve powerful performance $[16,17]$. The frequency-domain representation of FOPID controller is given by:

$$
C(s)=K_{p}+\frac{K_{I}}{s^{\lambda}}+K_{D} S^{\mu}
$$

where $K_{p}$ is the proportional gain, $K_{D}$ is a derivative gain, and $K_{I}$ is integral gain, and $(\lambda, \mu)$ is the fractional order of FOPID. In the case of $\lambda=1 \& \mu=1$, FOPID will act as a normal PID controller so that when $\lambda=0 \&$ $\mu=1$ will give a PD controller, and when $\lambda=1 \& \mu=0$ will give a PI controller.

\subsection{Standard whale optimization algorithm}

The standard WOA is a nature-inspired optimization algorithm invented by Mirjalili and Lewis [18], which based on bubble-net hunting of whales. This method used to mimic physical or biological phenomena, WOA is one of many other swarm-intelligence algorithms that invented in the last years, some of several famous algorithms are ray optimization algorithm [19], ant colony algorithm (ACO) [20], black hole algorithm [21]. The dominant point of this optimization method is that it merges the best individuals to create the next individuals. And since the whales are hunting in shrinking circle and on the spiral path, as explained in (17) below,

$$
\vec{X}(t+1)=\left\{\begin{array}{lll}
\vec{X}^{*}(t)-\vec{A} \cdot \vec{D} & \text { if } p<0.5 \\
\overrightarrow{D^{\prime}} \cdot e^{b l} \cdot \cos (2 \pi l)+\vec{X}^{*}(t) & \text { if } p \geq 0.5 & \text { (a) }
\end{array}\right.
$$

where (17a) represent the shrinking circle and (17b) defined the spiral path, $\boldsymbol{b}$ is a constant number for defining the shape of a logarithmic spiral, $l$ is a random number in the range $(-1,1), p$ is a random number between $[0,1], \overrightarrow{X^{*}}$ is the best solution for the position vector, and $\vec{A}$ is a coefficient vector and calculated as shown in the following formula,

$$
\vec{A}=2 \vec{a} \cdot \vec{r}-\vec{a}
$$

where $\vec{a}$ is a range number that decreased in the interval $(2,0), \vec{r}$ is a random vector of range $(1,0)$, and $\vec{D}$ can be defined as the global search and represented as shown in (19)

$$
\vec{D}=\left|\vec{C} \cdot \vec{X}_{\text {rand }}-\vec{X}\right|
$$


$\vec{C}$ is coefficient vector and can be defined from (20);

$$
\vec{C}=2 \cdot \vec{r}
$$

$\overrightarrow{D^{\prime}}$ is the interval distance between the whale and the prey which can be defined by (21),

$$
\overrightarrow{D^{\prime}}=\left|\vec{X}^{*}(t)-\vec{X}(t)\right|
$$

The humpback whale can locate their prey and encircle them this behavior represented as the following;

$$
\vec{D}=\left|C \cdot \vec{X}^{*}(t)-\vec{X}(t)\right|
$$

\section{IMPROVED WHALE OPTIMIZATION ALGORITHM}

The main problems in the standard WOA are its trail to escape from the large number of local solutions in nonlinear search spaces and the balancing issue between the exploration and exploitation. The procedure of the update is that when the algorithm updates the position in each iteration, the values of these updated values can reach a higher value. Values can reach beyond the upper bound and lower bound of the required functions. Mirjalili and Lewis [18], in (19) it proposed a random matrix-vector (random whale) to be chosen from each vector at every loop, as shown in the following statement,

$$
X_{\text {rand }}=\operatorname{Array}\left(\text { Integer }\left[\text { random }_{\text {number }}\right]\right)
$$

where, the $X_{\text {rand }}$ represent a random hunter (whale) vector that has only one value to be selected at each $A \geq$ 1 and this value might be not optimal value that make search domain converge to the prey for the shrinking circle in exploration phase and neglect the remaining values of $X_{\text {rand }}$ to solve this problem a search domain created to obtain the best value for (24),

$$
\overrightarrow{D_{\text {new }}}=\left|\vec{C} \cdot \vec{X}_{\text {best }}-\vec{X}\right|
$$

Let the developed position of the shrinking encircling step is h,

$$
\mathrm{h}=X_{\text {best }}-A * \overrightarrow{D_{\text {new }}}
$$

A comparison between the value of $h$ and the position of each search agent should be achieved; therefore, if new values of $h$ are smaller than the old position of search agent, it will be updated as a new position. To guarantees that the values of the position will be within the upper and lower bounds for the positive and negative values except when the value of position is positive, and the new value acquired from $h$ is negative so that the recently updated position will update the absolute $h$. To obtain better performance for the required benchmark functions and the system that will discuss in the next section, the following Algorithm 1 represents the running procedure of the IWOA,

Algorithm 1: The improved WOA

Input: The IWO algorithm external parameters.

Step 1: Specify the $L B, U B$ and dimension of the selected fitness function;

Step 2: Evaluate the fitness of each search agent and select the best one;

Step 3: Begin the main loop and for each search agent update the values of, interval range $(a)$, coefficient vector $(A, C)$, a random number $(L)$, and $(p)$;

Step 4: If $p$ less than $(0.5)$ and $(A)$ less than (1) then update the current position by (23);

Step 5: If $A$ greater than (1), calculate the value of. (25), and the value of $(h)$ using (26) based on the selection process of each search agent using (24);

Step 6: Check the current position if exceed the UB or LB define in Step 1, then replace it by another value $(h)$ such that the new value should be between the range of $(U B$, and $L B)$ otherwise return to Step 5 and choose a new value;

Step 7: If $p$ greater than or equal to $(0.5)$ then update the current position using $(18 \mathrm{~b})$;

Step 8: Repeat Step 3 until it reaches the maximum number of iterations;

Output: The optimum solution. 


\section{NUMERICAL RESULTS AND DISCUSSIONS}

\subsection{Test functions details}

Test functions can be classified into single local minima or by another meaning (unimodal), and many local minima, which is called multimodal and other functions to test the performance of the optimization algorithm, whether it is good to use or not with a comparison with other well-known optimization algorithms to prove its effectiveness. The benchmark functions are a set of six unimodal functions have used (F01-F06), while five multimodal functions have been used (F07-F11), and lastly, fixed dimension multimodal functions are used (F12-F15). The comparison is performed with gravitational search algorithm [22] and particle swarm optimization [23].

\subsection{Proposed algorithm evaluation}

The proposed algorithm has been tested based on the selected fifteen test functions and compared with the global optimization algorithms, namely, GSA and PSO. All test has been rerun 30 times with the maximum iterations of 500 and mean, and the standard deviation is calculated. Table 1 illustrates the results of the selected benchmark functions, while Figure 2. Show the IWOA performance versus other optimization algorithms for selected test functions (Sphere and Ackley functions).

Table 1. Comparison of IWOA with WOA, GSA and PSO algorithms

\begin{tabular}{ccccccccc}
\hline \multirow{2}{*}{ F } & \multicolumn{2}{c}{ IWOA } & \multicolumn{2}{c}{ WOA } & \multicolumn{2}{c}{ GSA } & \multicolumn{2}{c}{ PSO } \\
\cline { 2 - 8 } & mean & std & mean & std & mean & std & mean \\
F1 & $3.5275 \mathrm{E}-107$ & $1.4614 \mathrm{E}-106$ & $1.3091 \mathrm{E}-74$ & $6.8731 \mathrm{E}-74$ & 0.0278 & 0.1520 & $8.9207 \mathrm{E}-05$ & $1.0527 \mathrm{E}-04$ \\
F2 & 27.9086 & 0.5040 & 27.9863 & 0.4426 & 107.3099 & 112.7567 & 78.2038 & 68.5566 \\
F3 & 0.5883 & 0.3614 & 0.4254 & 0.2013 & 13.2667 & 19.8319 & $2.4612 \mathrm{E}-04$ & $4.3406 \mathrm{E}-04$ \\
F4 & $9.5657 \mathrm{E}-26$ & $5.2334 \mathrm{E}-25$ & $4.4861 \mathrm{E}+04$ & $1.3727+\mathrm{E} 04$ & 971.1853 & 319.8463 & 89.5326 & 29.7383 \\
F5 & $1.1230 \mathrm{E}-25$ & $3.0857 \mathrm{E}-25$ & 46.2694 & 31.2569 & 8.0052 & 2.5682 & 1.0902 & 0.2153 \\
F6 & 0 & 0 & 0 & 0 & 29.8819 & 9.4226 & 60.3496 & 14.8764 \\
F7 & $1.2434 \mathrm{E}-15$ & $1.0840 \mathrm{E}-15$ & $3.9672 \mathrm{E}-15$ & $2.4210 \mathrm{E}-15$ & $1.1954 \mathrm{E}-08$ & $2.4148 \mathrm{E}-09$ & 0.1354 & 0.3701 \\
F8 & 0 & 0 & $3.7896 \mathrm{E}-15$ & $1.4422 \mathrm{E}-14$ & 29.3181 & 7.3381 & 53.1311 & 11.0473 \\
F9 & 0 & 0 & 0.0040 & 0.0217 & 25.9758 & 6.4710 & 0.0067 & 0.0086 \\
F10 & 0.0314 & 0.0219 & 0.0487 & 0.1270 & 1.5966 & 0.8672 & 0.0207 & 0.0633 \\
F11 & 0.0019 & 0.005 & 0.006329 & 0.003765 & 0.0049 & 0.0039 & 0.009236 & 0.0010973 \\
F12 & -7.3048 & 2.7766 & -6.6013 & 3.3175 & -10.1891 & 1.713 & -8.7908 & 2.7718 \\
F13 & -8.2746 & 2.4917 & -6.5921 & 2.8524 & -6.4268 & 3.6015 & 3.6015 & 3.6015 \\
F14 & -3.1992 & 0.1160 & -3.1851 & 0.1253 & -3.3179 & 0.0225 & -3.2625 & 0.0605 \\
\hline
\end{tabular}

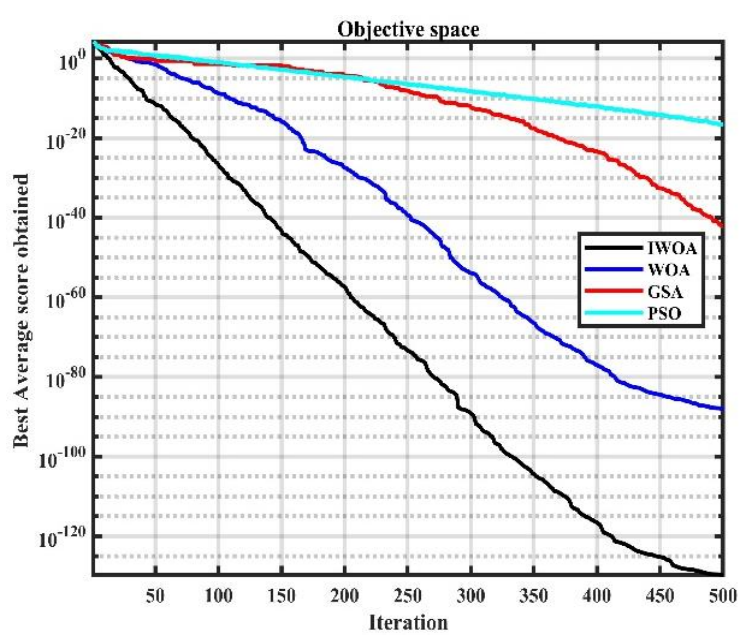

(a)

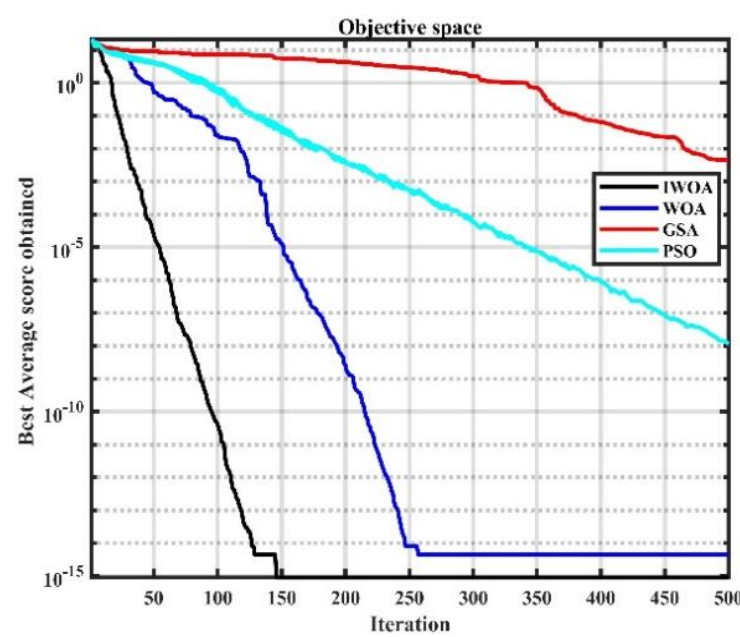

(b)

Figure 2. Algorithm's performance comparison based on the selected test functions,

(a) F1 (Sphere function), (b) F8 (Ackley function)

As aforesaid, the unimodal test functions have only one local minima value while multimodal test functions have many local minima values increased by increasing the dimension number of the tasks. However, from the result achieved above, it is evident that unimodal test functions (F1, F4, F5 \& F6), 
multimodal test functions (F7, F8 \& F10) tend to reach towards more optimal minimum value than that WOA. At the same time, it almost converges in (F2 \& F3) and in fixed dimension multimodal function (F12), but they have a lower minimum amount than WOA, (F11, F13 \& F14) are equally or less converges due to the nature of mathematical tasks used, which has a limited dimension range. Knowing that, each function was executed for 30 times of with 500 iterations to obtain the best average and standard deviation.

\subsection{AUV model results and discussions}

A mathematical model of AUV designed in Simulink platform as state-space block with a block diagram of the LQG controller, an observer with desired eigenvalues and noise power is depicted in Figure 3. While the band-limited white noise that utilized to examine the developed system in this research work is with (0.00001) power and (0.01) sample time, where the main research work objective is to remove the effect of this noise to enhance the AUV's work. A step input is considered as input in the overall proposed system shown in Figure 3.

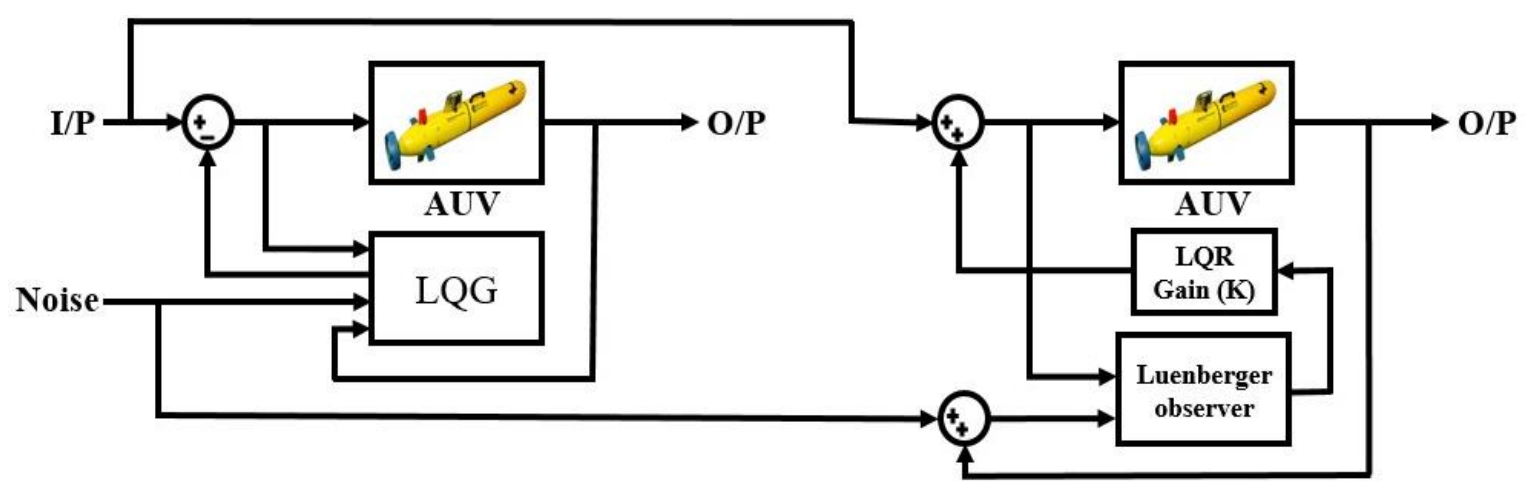

Figure 3. The overall proposed closed-loop system

The proposed FOPID controller based on basic and improved algorithms depicted in Figure 4, where the objective functions that used to evaluate the performance index (PI) are integrated time absolute error (ITAE), integrated absolute error (IAE), and integrated squared error (ISE). Because of evolving additive white noise, the response of AUV vertical movement manages to be distorted and that evident in the yellow curve denoted the output of Luenberger observer (distorted signal). In addition, LQG signal is obtained that represented in the blue curve alongside with FOPID based WOA and IWOA algorithms are illustrated in Figure 5(a), while Figure 5(b) shows the distort signal, LQG signal, and the PID response based on WOA and IWOA algorithms when the noise power is increased to (0.001). In contrast, for each curve, other results are obtained based on time specifications (rising time, settling time, and maximum peak overshoot), as shown in Table 2 [24-27] when the first noise power (0.00001) is used, while Table 3 shows the time specifications when the noise power is increased to (0.001). The stopping criteria of the optimization algorithms depending on reaching a specific tolerance, instantly the simulation will stop with the most suitable characteristics of the system response.

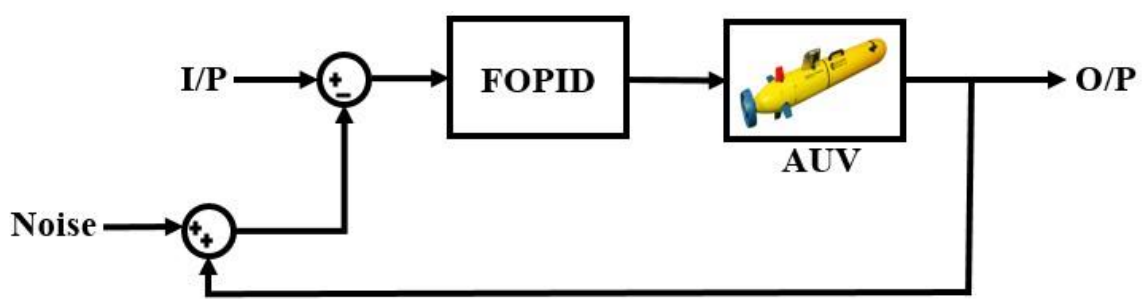

Figure 4. Proposed FOPID tuned by standard and improved WOA 


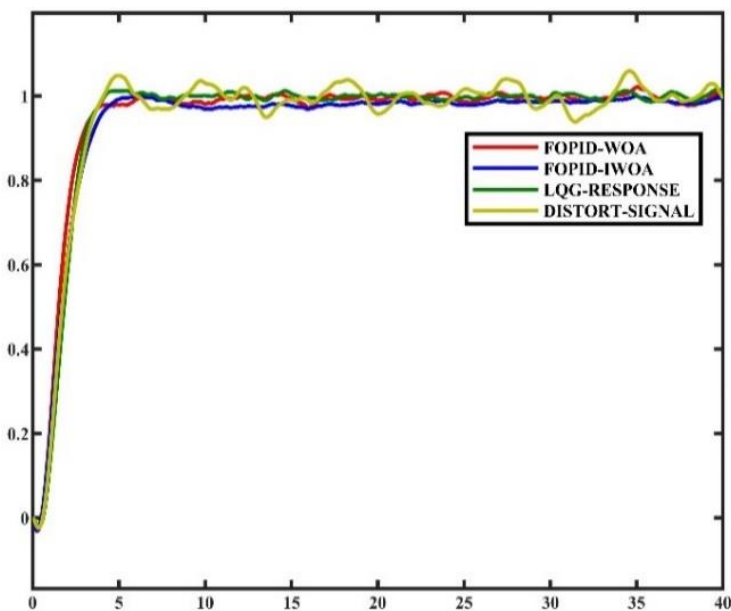

(a)

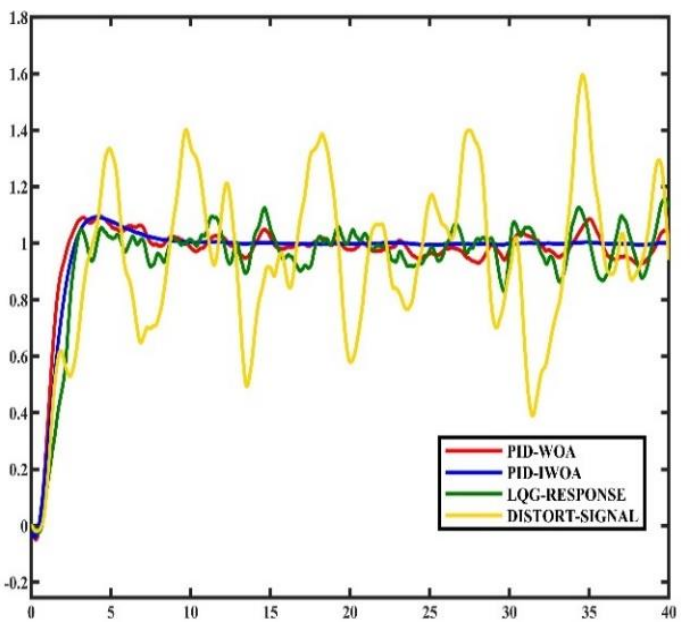

(b)

Figure 5. The system response based on different controllers and optimization algorithms with distortion, (a) FOPID controller, (b) PID controller

Table 2. Characteristics of output signal (Luenberger observer (distort signal), LQG, FOPID-IWOA, and FOPID-WOA)

\begin{tabular}{|c|c|c|c|c|c|c|c|c|c|c|c|c|}
\hline \multirow{3}{*}{ Specifications } & \multirow{3}{*}{$\begin{array}{l}\text { Distort } \\
\text { signal }\end{array}$} & \multirow{3}{*}{ LQG } & \multicolumn{5}{|c|}{ FOPID-IWOA } & \multicolumn{5}{|c|}{ FOPID-WOA } \\
\hline & & & $\mathrm{Kp}$ & $\mathrm{Ki}$ & $\mathrm{Kd}$ & $\mu$ & $\lambda$ & $\mathrm{Kp}$ & $\mathrm{Ki}$ & $\mathrm{Kd}$ & $\mu$ & $\lambda$ \\
\hline & & & 0.1051 & 0.0376 & 0.3194 & 0.9929 & 0.9993 & 0.1036 & 0.0422 & 0.3587 & 1 & 0.9024 \\
\hline Rising time & 2.2688 & 2.1465 & \multicolumn{5}{|c|}{2.1460} & \multicolumn{5}{|c|}{0.9720} \\
\hline P.O \% & 6.5937 & 0.2946 & \multicolumn{5}{|c|}{0.6260} & \multicolumn{5}{|c|}{6.9846} \\
\hline peak & 1.0597 & 1.0158 & \multicolumn{5}{|c|}{1.0083} & \multicolumn{5}{|c|}{1.0706} \\
\hline Peak time & 34.5797 & 39.7279 & \multicolumn{5}{|c|}{34.9547} & \multicolumn{5}{|c|}{2.4363} \\
\hline
\end{tabular}

From the result obtained in Table 2, it is evident that FOPID-IWOA has the lowest settling time between FOPID-WOA, and LQG while the lowest rising time take place in FOPID-WOA. The nearest point to step input and peak time can be seen in FOPID-IWOA followed by LQG, distort signal, FOPID-WOA; while the minimum overshoot spotted in ascending order in LQG, FOPID-IWOA, distort signal, FOPIDWOA. Table 3 shows the system response of different controllers when noise is increased with their characteristics.

Table 3. Characteristics of the output signal (Luenberger observer (distort signal), LQG, PID-IWOA, and

\begin{tabular}{|c|c|c|c|c|c|c|c|c|}
\hline & & & & (OA) & & & & \\
\hline \multirow{3}{*}{ Specifications } & \multirow{3}{*}{ Distort signal } & \multirow{3}{*}{ LQG } & \multicolumn{3}{|c|}{ PID-IWOA } & \multicolumn{3}{|c|}{ PID-WOA } \\
\hline & & & $\mathrm{Kp}$ & $\mathrm{Ki}$ & $\mathrm{Kd}$ & $\mathrm{Kp}$ & $\mathrm{Ki}$ & $\mathrm{Kd}$ \\
\hline & & & 0.1476 & 0.0583 & 0.3621 & 0.1592 & 0.0696 & 0.3688 \\
\hline Rising time & 2.3625 & 1.9773 & & 1.6312 & & & 1.3755 & \\
\hline Settling time & 39.9771 & 39.8559 & & 7.3444 & & & 39.4393 & \\
\hline P.O \% & 69.7015 & 2.6361 & & 8.9876 & & & 4.4435 & \\
\hline peak & 1.5961 & 1.1572 & & 1.0928 & & & 1.0960 & \\
\hline Peak time & 34.5800 & 39.7300 & & 4.2019 & & & 4.2753 & \\
\hline
\end{tabular}

From the result obtained in Table 3, it is clear that PID-IWOA has the lowest settling time between PIDWOA and LQG while the lowest rising time toke place in PID-WOA. The nearest point to step input and peak time can be seen in PID-IWOA followed by PID-WOA, LQG, distort signal. At the same time, the minimum overshoot spotted in ascending order in LQG, PID-WOA, PID-IWOA, distort signal.

\section{CONCLUSION}

AUV model is formed based on linearized vertical motion control, and due to the noise, that caused by sensors or low turbulence on the AUV, that has to lead to distorting the response such as (rising time, 
settling time, and overshoot) of the AUV which establishes as the main problem. Different controlling schemes were used like LQG, PID and FOPID that tuned based on standard and improved WOA. The proposed IWOA was tested, and the results are compared with other swarm algorithms using different test functions (unimodal, multimodal and fixed dimensions multimodal). IWOA showed that it tends to reach optimal minimum value that smaller than WOA and outperform on other natural-inspired algorithms.

Simulation results for the AUV's system are obtained and compared for the controllers used as an example, the rising time of FOPID-IWOA is lower than LQG, lower than Luenberger observer (distort signal), while the settling time lower than LQG, and lower than distort the signal. At the same time, the rising time of PID-IWOA is lower than LQG, lower than Luenberger observer (distort signal) while the settling time lower than LQG, and lower than distort the signal. Finally, the performance of the system was enhanced, but at the same time, it took a higher number of iterations to reach these features.

\section{ACKNOWLEDGEMENTS}

We would like to express our gratitude and thanks to the editors of BEEI journal and the anonymous reviewers for their valuable instructions and notifications.

\section{REFERENCES}

[1] R. Wiryadinata, A. S. Nurliany, I. Muttakin, and T. Firmansyah, "Design of a low cost remotely operated vehicle with 3 DoF navigation," Bulletin of Electrical Engineering and Informatics, vol. 6, no. 1, pp. 13-23, 2017.

[2] M. I. Sani, S. Siregar, A. P. Kurniawan, and M. A. Irwan, "FIToplankton: Wireless controlled remotely-operated underwater vehicle (ROV) for shallow water exploration," International Journal of Electrical and Computer Engineering ,vol. 8, no. 5, pp. 3325-3332, 2018.

[3] S. Siregar, M. I. Sani, M. M. Kurnia, and D. Hasbialloh, "Low-cost communication system for explorer-class underwater remotely operated vehicle," Telecommunication, Computing, Electronics and Control, vol. 17, no. 2, pp. 593-600, 2019.

[4] S. A. Rahmah, E. H. Binugroho, R. S. Dewanto, and D. Pramadihanto, "Velocity control of ROV using modified integral SMC with optimization tuning based on Lyapunov analysis," Telecommunication, Computing, Electronics and Control, vol. 18, no. 3, pp. 1505-1513, 2020.

[5] S. Siregar, M. I. Sani, and S. T. Parlindungan Silalahi, "Single camera depth control in micro class ROV," Telecommunication, Computing, Electronics and Control, vol. 18, no. 3, pp. 1546-1552, 2020.

[6] Z. Li, W. Liu, L. Gao, L. Li, and F. Zhang, "Path planning method for AUV docking based on adaptive quantumbehaved particle swarm optimization," IEEE Access, vol. 7, pp. 78665-78674, 2019.

[7] Y. Ma, Y. Gong, C. Xiao, Y. Gao and J. Zhang, "Path planning for autonomous underwater vehicles: an ant colony algorithm incorporating alarm pheromone," IEEE Transactions on Vehicular Technology, vol. 68, no. 1, pp. 141154, Jan. 2019.

[8] Xin Yao, Yong Liu, and Guangming Lin, "Evolutionary programming made faster," IEEE Transactions on Evolutionary Computation, vol. 3, no. 2, pp. 82-102, July 1999.

[9] X. S. Yang, "Firefly algorithm, stochastic test functions and design optimization," International Journal of BioInspired Computation, vol. 2, no. 2, pp. 78-84, 2010.

[10] M. Molga and C. Smutnicki, "Test functions for optimization needs," Test Functions for Optimization Needs, vol. 101, no. 48, pp. 1-43, 2005.

[11] C. Shen, Y. Shi, and B. Buckham, "Lyapunov-based model predictive control for dynamic positioning of autonomous underwater vehicles," 2017 IEEE International Conference on Unmanned Systems (ICUS), pp. 588593, 2017.

[12] N. Fischer, S. Bhasin, and W. E. Dixon, "Nonlinear control of an autonomous underwater vehicle: A RISE-based approach," Proceedings of the 2011 American Control Conference, pp. 3972-3977, 2011.

[13] T. Prestero, "Verification of a six-degree of freedom simulation model," Thesis, Dept. of Ocean Engineering; and the Woods Hole Oceanographic Institution); and, (S.M.)--Joint Program in Applied Ocean Science and Engineering (Massachusetts Institute of Technology, Dept. of Mechanical Engineering; and the Woods Hole Oceanographic Institution), Massachusetts Institute of Technology, 2001.

[14] J. Zarei, A. Montazeri, M. Reza Jahed Motlagh, and J. Poshtan, "Design and comparison of LQG/LTR and Ho controllers for a VSTOL flight control system," Journal of the Franklin Institute, vol. 344, no. 5, pp. 577-594, 2007.

[15] R. Soni and Sathans, "Optimal control of a ball and beam system through LQR and LQG," 2018 2nd International Conference on Inventive Systems and Control (ICISC), pp. 179-184, 2018.

[16] O. W. Abdulwahhab and N. H. Abbas, "A new method to tune a fractional-order PID controller for a twin rotor aerodynamic system," Arabian Journal for Science and Engineering, vol. 42, no. 12, pp. 5179-5189, 2017.

[17] O. W. Abdulwahhab and N. H. Abbas, "A new analytic method to tune a fractional order PID controller," Journal of Engineering, vol. 23, no. 12, pp. 1-12, 2017.

[18] S. Mirjalili and A. Lewis, "The whale optimization algorithm," Advances in Engineering Software, vol. 95, pp. 5167, 2016.

[19] A. Kaveh and M. Khayatazad, "A new meta-heuristic method: ray optimization," Computers \& Structures, vol. 
112, pp. 283-294, 2012.

[20] M. Dorigo, M. Birattari, and T. Stutzle, "Ant colony optimization," IEEE Computational Intelligence Magazine, vol. 1 , no. 4, pp. 28-39, Nov. 2006.

[21] A. Hatamlou, "Black hole: A new heuristic optimization approach for data clustering," Information Sciences, vol. 222, pp. 175-184, 2013.

[22] E. Rashedi, H. Nezamabadi-pour, and S. Saryazdi, "GSA: A gravitational search algorithm," Information Sciences, vol. 179, no. 13, pp. 2232-2248, 2009.

[23] J. Kennedy and R. Eberhart, "Particle swarm optimization," Proceedings of ICNN'95-International Conference on Neural Networks, vol. 4, pp. 1942-1948, 1995.

[24] R. A. Krohling and J. P. Rey, "Design of optimal disturbance rejection PID controllers using genetic algorithms," IEEE Transactions on Evolutionary Computation, vol. 5, no. 1, pp. 78-82, Feb. 2001.

[25] T. Kawabe and T. Tagami, "A real coded genetic algorithm for matrix inequality design approach of robust PID controller with two degrees of freedom," Proceedings of 12th IEEE International Symposium on Intelligent Control, pp. 119-124, 1997.

[26] Y. Mitsukura, T. Yamamoto, and M. Kaneda, "A design of self-tuning PID controllers using a genetic algorithm," Proceedings of the 1999 American Control Conference (Cat. No. 99CH36251), vol. 2, pp. 1361-1365, 1999.

[27] N. H. Abbas, "Tuning of different controlling techniques for magnetic suspending system using an improved bat algorithm," International Journal of Electrical and Computer Engineering, vol. 10, no. 3, pp. 2402-2415, 2020.

\section{BIOGRAPHIES OF AUTHORS}

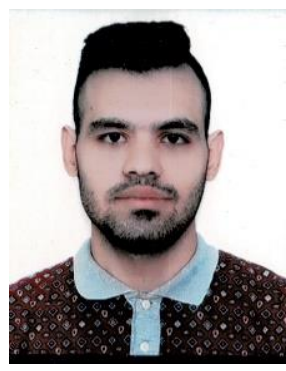

Mustafa Wassef was born in Baghdad, 1992. He is a M.Sc research scholar in computer and control specialization with B.Sc. degree in Electrical Engineering from University of Baghdad, Baghdad, Iraq in 2017, and a Technical Diploma in electrical power from Middle Technical University, Baghdad, Iraq, in 2012. His research interests include controllers design, AUV modeling \& control and swarm intelligence optimization techniques.

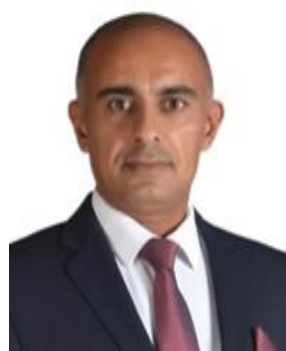

Nizar Hadi Abbas was born in Baghdad, 1975. He received his B.Sc. degree in electrical engineering from University of Baghdad, Baghdad, Iraq in 2000, a M.Sc. degree in control and computer engineering in 2002 from University of Baghdad, and a Ph.D. degree in advanced control in 2011 from Osmania University, Hyderabad, India. He is presently an assistant professor. His research interests include control theory, controllers design, fractional order systems, robot modeling \& control and modern optimization techniques. He published around 30 scientific articles. 